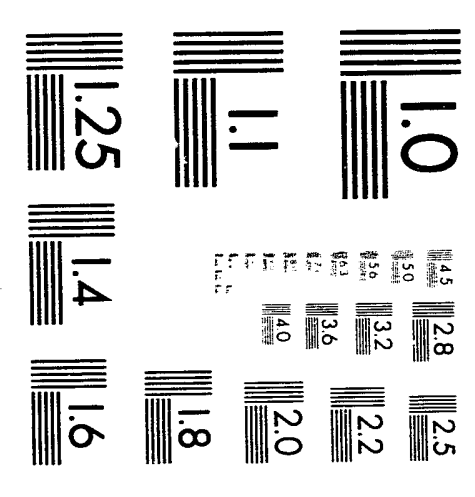



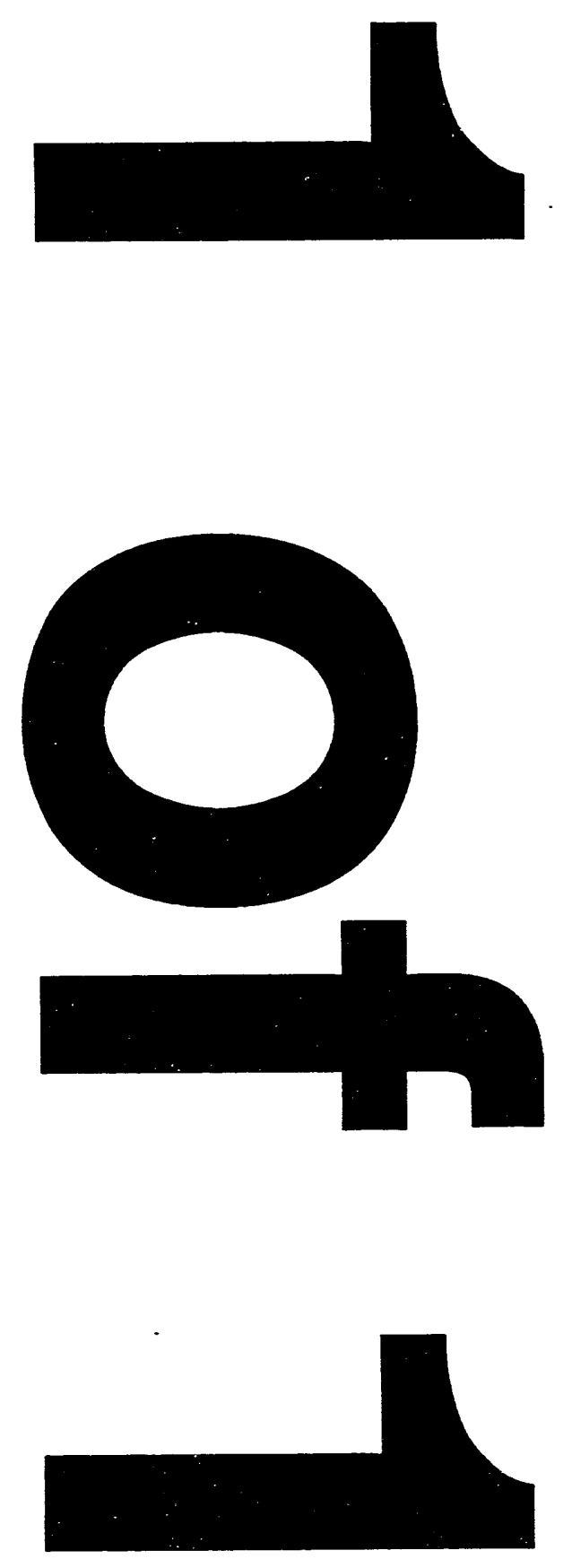


\title{
Parallel Mathematical Software
}

\author{
Annual Report \\ February 15, 1990 - August 14, 1991 \\ Layne T. Watson and Calvin J. Ribbens \\ Departments of Computer Science and Mathematics \\ Virginia Polytechnic Institute and State University \\ Blacksburg, VA 24061-0106
}

January 31, 1991

\section{DISCLAIMER}

\begin{abstract}
This report was prepared as an account of work sponsored by an agency of the United States Government. Neither the United States Government nor any agency thereof, nor any of their employees, makes any warranty, express or implied, or assumes any legal liability or responsibility for the accuracy, completeness, or usefulness of any information, apparatus, product, or process disclosed, or represents that its use would not infringe privately owned rights. Reference herein to any specific commercial product, process, or service by trade name, trademark, manufacturer, or otherwise does not necessarily constitute or imply its endorsement, recommendation, or favoring by the United States Government or any agency thereof. The views and opinions of authors expressed herein do not necessarily state or reflect those of the United States Government or any agency thereof.
\end{abstract}

Prepared for the U.S. Department of Energy under Grant DE-FG05-88ER25068. 


\section{Annual Report on DOE Grant DE-FG05-88ER25068}

\section{Parallel Mathematical Software}

\section{Parallel ELLPACK.}

We review relevant work on parallel ELLPACK under DOE Grant DE-FG05-88ER25068 in three major areas: shared memory parallel ELLPACK, domain decomposition algorithms, and parallel scientific computing environments.

Shared memory parallel ELLPACK. A version of ELLPACK targeted specifically for shared memory parallel machines has been designed and is being implemented on a Sequent Symmetry S81 computer. This version of ELLPACK contains a representative selection of the problem solving modules in the original ELLPACK system (approximately 15 modules), as well as several new modules, including domain decomposition methods and a GMRES iterative solver. The design of shared memory parallel ELLPACK is discussed in

C.J. Ribbens, "On parallel ELLPACK for shared memory machines", Department of Computer Science Report TR 90-46, VPI \& SU, 1990.

A conference presentation in this area is

C. J. Ribbens, "Parallel ELLPACK for shared-memory machines", SIAM Annual Meeting, Chicago, July, 1990.

Domain decomposition. Our work has concentrated on the implementation and performance of Schwarz-splitting algorithms on realistic problems and on real machines. A better understanding of the influence of memory paradigm (shared vs. distributed), of scalability, and of the usefulness of parallel programming tools for such algorithms has been achieved. We have also studied domain decomposition methods which employ simple adaptive grid schemes in determining a good decomposition. The influence of adaptation on the domain decomposition approach, particularly on the performance of preconditioners, is of interest. Results in this area are described in

J. Gamble and C.J. Ribbens, "Performance comparison of three parallel implementations of a Schwarz splitting algorithm", Department of Computer Science Report TR 89-37, VPI \& SU, 1989;

C. J. Ribbens and L. T. Watson, "The parallel performance of Schwarz splitting", Department of Computer Science Report TR 90-34, VPI \& SU, 1990;

C.J. Ribbens, "A moving mesh scheme for adaptive domain decomposition", Proceedings of the ICASE Symposium on Unstructured Scientific Computation on Scalable Multiprocessors, submitted.

Relevant conference presentations include

C. J. Ribbens, "Schwarz splitting in ELLPACK", Virginia Tech-ICAM Conference on Numerical Solutions of Partial Differential Equations, Blacksburg, VA, September, 1988;

C. J. Ribbens and L. T. Watson, "Parallel performance of Schwarz splitting", SIAM Conference on Domain Decomposition Methods, Houston, March, 1989;

C. J. Ribbens and L .T. Watson, "Shared memory vs. distributed memory for Schwarz splitting", SIAM Annual Meeting, San Diego, July, 1989;

C. J. Ribbens, "A moving mesh scheme for dynamically adaptive domain decomposition", ICASE Workshop on Unstructured Scientific Computation on Scalable Multiprocessors", Kill Devil Hill, NC, October, 1990; 
C. J. Ribbens, "On adaptive domain decomposition with moving subdomains", Fifth Conference on Domain Decomposition Methods for Partial Differential Equations, Norfolk, May, 1991, suhmitted.

Parallel scientific computing environments. Two important aspects of effectively using a modern heterogencous computing environment have been studied: parallel programming languages and interactive user interfaces. Results in this area are reported in:

J. Gamble, "Explicit Parallel Programming", M.S. thesis, Department of Computer Science, VPI \& SU, 1990;

J. Gamble and C.J. Ribbens, "Explicit parallel programming: User's guide", Department of Computer Science Report TR 91-3, VPI \& SU, 1991;

J. Gamble and C.J. Ribbens, "Explicit parallel programming: System description", Department of Computer Science Report TR 91-4, VPI \& SU, 1991;

J. Gamble and C.J. Ribbens, "Explicit parallel programming", manuscript.

Relevant conference presentations include:

J. Gamble, "Explicit parallel programming", Virginia Computer Users Conference, Blacksburg, September, 1990;

C. deSa and C.J. Ribbens, "An interactive problem solving environment for domain decomposition", Second International Conference on Industrial and Applied Mathematics, Washington, D.C., July 1991, accepted.

\section{Parallel HOMPACK.}

Nonlinear systems of equations can be roughly classified into one of three types: 1) small polynomial systems for which all solutions are sought; 2 ) small systems of order up to several hundred, where the Jacobian matrix can be treated as full; and 3) large systems whose Jacobian matrices are sparse, and the sparse matrix structures must be dealt with explicitly. Other possibilities such as large polynomial systems or large dense systems are intractable, and will not be considered further. There are always intermediate size problems, and whether these are treated as type 2) or type 3 ) is frequently a matter of algorithmic choice or available computer technology. HOMPACK contains several homotopy algorithms for problems in each of classes 1), 2), and 3).

The serial homotopy algorithms for types 1) and 2) are well known and generally accepted. Sparse matrix technology is an active research area, however, so the HOMPACK sparse matrix codes for type 3) problems are by no means the best avinilable, although there is evidence that the HOMPACK serial sparse codes are competitive. Progress under the current DOE Grant DEFG05-88ER25068 on parallel code for each of the three problem types will be described in turn.

Parallel algorithms for polynomial systems have been extensively studied by the PI's, their colleagues, and their students. Computer code has been developed and tested for the parallel shared memory machines Encore Multimax, Alliant FX/8, Elxsi 6400, Sequent Symmetry S81, and the distributed memory Intel $\mathrm{iPSC} / 1$ hypercube. This work resulted in a Ph.D. thesis

Amal Chakraborty, "Parallel homotopy curve tracking on a hypercube", Ph.D. thesis, Dept. of Computer Sci., VPI \& SU, Blacksburg, 1990;

and the conference and journal papers

A. P. Morgan and L. T. Watson, "A globally convergent paraliel algorithm for zeros of polynomial systems", Nonlinear Anal., 13 (1989) 1339-1350; 
W. Pelz and L. T. Watson, "Message length effects for solving polynomial systems on a hypercube", Parallel Comput., 10 (1989) 161-176;

A. P. Morgan, A. J. Sommese, and L. T. Watson, "Finding all isolated solutions to polynomial systems using HOMPACK", ACM Trans. Math. Software, 15 (1989) 93-122;

D. C. S. Allison, S. Harimoto, and L. T. Watson, "The granularity of parallel homotopy algorithms for polynomial systems of equations", Internat. J. Comput. Math., 29 (1989) 21-37;

D. C. S. Allison, A. Chakraborty, and L. T. Watson, "Granularity issues for solving polynomial systems via globally convergent algorithms on a hypercube", J. Supercomputing, 3 (1989) $5-20$;

L. T. Watson and A. P. Morgan, "Serial and parallel global optimization of polynomial programs via homotopy algorithms", SIAM J. Optim., submitted;

D. C. S. Allison, A. Chakraborty, and L. T. Watson, "Granularity issues for solving polynomial systems via globally convergent algorithms on a hypercube", in Proc. Third Conf. on Hypercube Concurrent Computers and $\Lambda$ pplications, G. C. Fox (ed.), ACM, Pasadena, CA, 1988, 1463-1472;

D. C. S. Allison, S. Harimoto, and L. T. Watson, "The granularity of parallel homotopy algorithms for polynomial systems of equations", in Proc. 1988 Internat. Conf. on Parallel Processing, Vol. III, D. H. Bailey (ed.), St. Charles, IL, 1988, 165-168;

S. Harimoto and L. T. Watson, "The granularity of homotopy algorithms for polynomial systems of equations", in Parallel Processing for Scientific Computing, G. Rodrigue (ed.), SIAM, Philadelphia, PA, 1989, 115-120.

For the case of polynumial systems, the theory, algorithms, and serial computer code (POLSYS in HOMPACK) are all in good shape. Reasonably good parallel computer codes now exist for both shared memory and distributed memory multiprocessors. The original intent was to develop production quality parallel homotopy code, comparable to the serial HOMPACK code. However, the absence of a truly portable parallel language, or even an emerging standard, makes it clear that this goal is premature. Currently, the most prudent course would appear to be to develop parallel mathematical algorithms independent of any machine architecture, yet guided by the general constraints of the shared memory or distributed memory paradigm.

Parallelism for small dense nonlinear systems of equaticas is much more difficult to cxploit. For homotopy curve tracking, there are only three potential sources of parallelism: the algorithm logic, the linear algebra, and the function/derivative evaluations. The parallelism in the algorithm logic appears negligible. Both the linear algebra and function evaluation questions are tough. On the Sequent S81, the overhead cost of setting up a DOACROSS for a matrix multiply is greater than the cost of just doing it serially for small matrices $(n \approx 10)$, and on the iPSC/1 the communication cost outweighs the savings until $n>50$. Similarly function/derivative evaluations must be sufficiently costly to justify splitting them across processors. There are subtle interactions too-on the iPSC, if it pays to parallelize the Jacobian matrix evaluation, then the linear aigebra should also be done in parallel, even if the linear algebra done serially is faster. The reason is that the cost of collecting the matrix components into a single processor for serial linear algebra, and then doing that linear algebra serially, is (usually) more expensive than leaving the matrix components distributed and doing the linear algebra in parallel. For the iPSC hypercube, a comprehensive set of experiments was conducted comparing data decomposition strategies, different linear algebra 
algorithms, the effect of function evaluation cost and probabilistic distribution, parallel paradigms, and the interaction of all these factors. The results have been reported in

Amal Chakraborty, "Parallel homotopy curve tracking on a hypercube", $\mathrm{Ph} . \mathrm{D}$. thesis, Dept. of Computer Sci., VPI \& SU, Blacksburg, 1990;

A. Chakraborty, D. C. S. Allison, C. J. Ribbens, and L. T. Watson, "Note on unit tangent vector computation for homotopy curve tracking on a hypercube", Parallel Comput., submitted;

A. Chakraborty, D. C. S. Allison, C. J. Ribbens, and L. T. Watson, "Analysis of function component complexity for hypercube homotopy algorithms", IEEE Trans. Parallel Distrib. Sys., submitted;

A. Chakraborty, D. C. S. Allison, C. J. Ribbens, and L. T. Watson, "Parallel orthogonal decompositions of rectangular matrices for curve tracking on a hypercube", in Proc. Fourth Conf. on Hypercubes, Concurrent Computers, and Applications, J. L. Gustafson (ed.), ACM, Monterey, CA, 1989, 651-654;

A. Chakraborty, D. C. S. Allison, C. J. Ribbens, and L. T. Watson, "Parallel unit tangent vector computation for homotopy curve tracing on a hypercube", in Proc. 1990 AC'M Lighteenth Annual Computer Science Conf., Washington, DC, 1990, 103-108;

A. Chakraborty, D. C. S. Allison, C. J. Ribbens, and L. T. Watson, "Low dimensional homotopy curve tracking on a hypercube", in Proc. 1990 Internat. Conf. on Parallel Processing, Vol. III, P.-C. Yew (ed.), St. Charles, IL, 1990, 44-51;

A. Chakraborty, D. C. S. Allison, C J. Ribbens, and L. T. Watson, "Parallel homotopy curve tracking on a hypercube", in Parallel Processing for Scientific Computing, J. Dongarra, P. Messina, D. C. Sorensen, and R. G. Voigt (eds.), SIAM, Philadelphia, PA, 1990, 149-153;

L. T. Watson, "Numerical analysis of nonlinear equations in computer vision and robotics", in Numerical Linear Algebra, Digital Signal Processing and Parallel Algorithms, NATO ASI Series F, G. Golub and P. Van Dooren (eds.), Springer-Verlag, Berlin, 1990, 695-704.

For the code in HOMPACK addressing type 2) problems, a parallel version for the $\mathrm{PPSC} / 1$ (the harder case, compared to the Sequent) has been developed. Parallel code for shared memory machines (e.g., Sequent) remains to be developed.

Type 3) problems, and the concomitant sparse matrix technology, have received much of the principal investigators' attention during the current grant. Convergence theory, parallel algorithms, and practical implementation details have all received attention-sparse matrices are likely to remain a research interest of the principal investigators for some time to come. This work has resulted in a M.S. thesis, two solid SIAM journal articles, and several conference papers:

Kashmira Irani, "Preconditioned sequential and parallel conjugate gradient algorithms for homotopy curve tracking", M.S. thesis, Dept. of Computer Sci., VPI \& SU, Blacksburg, 1990;

K. M. Irani, M. P. Kamat, C. J. Ribbens, H. F. Walker, and I. T. Watson, "Experiments with conjugate gradient algorithms for homotopy curve tracking", SIAM J. Optim., to appear;

C. deSa, K. M. Irani, C. J. Ribbens, L. T. Watson, and H. F. Walker, "Preconditioned iterative methods for homotopy curve tracking", SIAM J. Sci. Stat. Comput., to appear;

K. M. Irani, C. J. Ribbens, and L. T. Watson, "Parallel HOMPACK: a case study in parallel mathematical software", Proc. Fifth SIAM Conf. on Parallel Processing for Scientific Computing, Houston, March, 1991; 
D. Žigić, E. G. Collins, S. Richter, and L. T. Watson, "A homotopy method for solving optimal projection equations for the reduced order model problem", IEEE Southeastcon, Williamsburg, VA, April, 1991.

Collaboration with Robert Melville and Ljiljana Trajkovic of AT\&T Bell Laboratories on the large nonlinear systems of equations arising in circuit simulation has had a significant impact. AT\& T is now developing a large circuit simulation code based on HOMPACK, and is supplying the DOE project with realistic large $(n>10,000)$ test problems. These sparse nonlinear systems range in size from $n=20$ to $n>\{0,000$, und have essentially random sparsity patterns. Typical matrix iterative methods developed for solving discretizations of PDEs have been woefully inadequate on the circuit simulation problems. It is clear that both parallelism and new sparse matrix algorithms will be required to solve realistic large circuit simulation problems. This collaboration with AT\&T is ongoing, has produced significant results some of which are embodied in

R. C. Melville, Lj. Trajković, S.-C. Fang, and L. T. Watson, "Globally convergent homotopy methods for the DC operating point problem", SIAM J. Optim., submitted, and will continue to benefit the proposed work. 

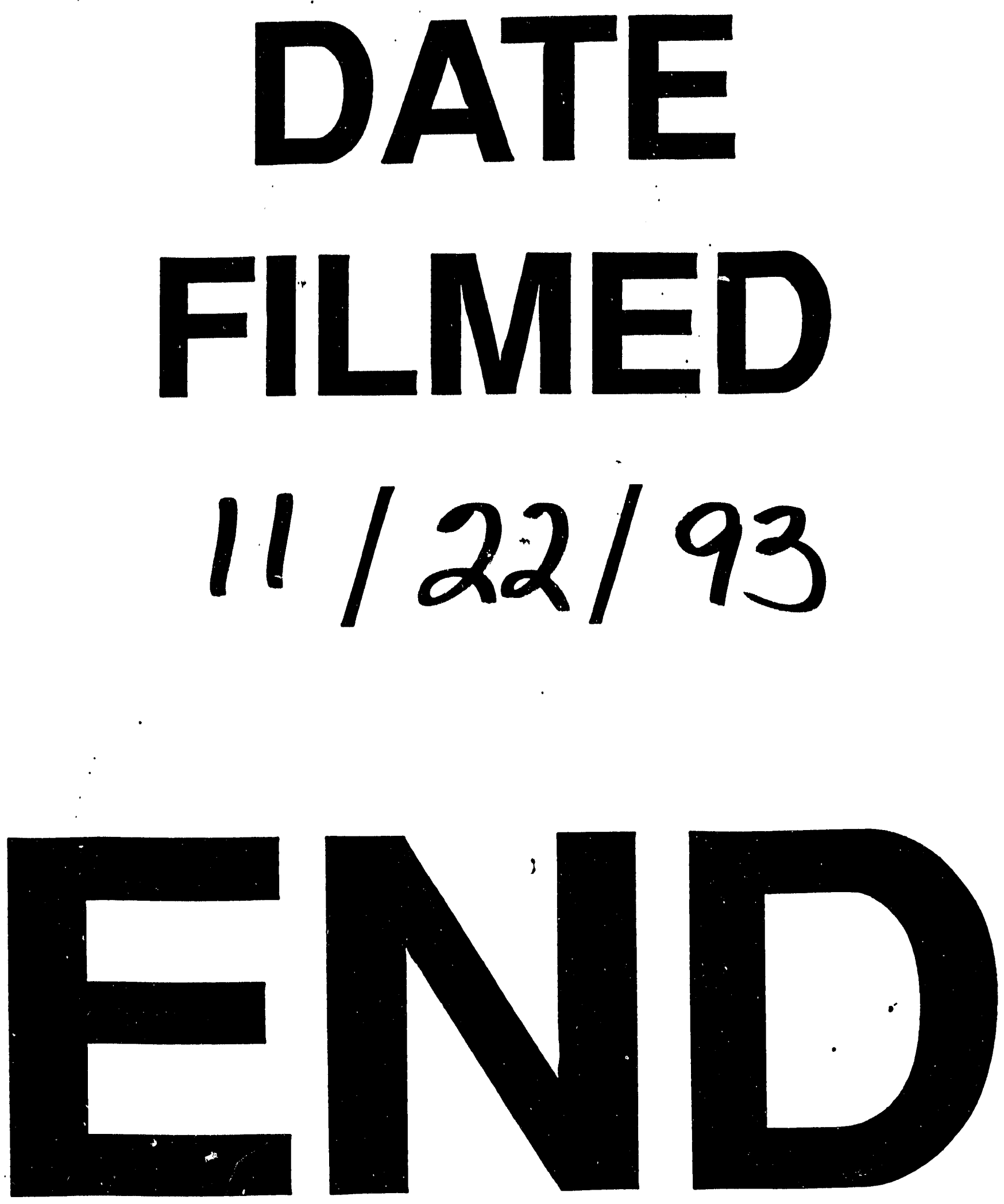
-

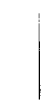

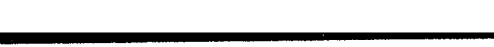

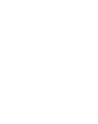

r

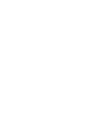

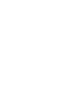

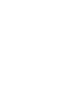

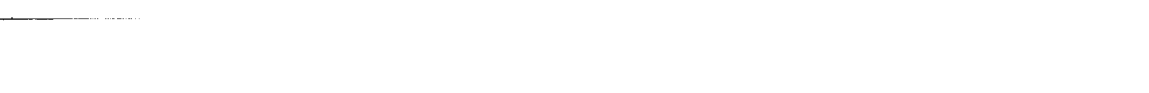

